\title{
Towards an Automatic Extraction of Generalized System of Contradictions Out of Solutionless Design of Experiments
}

\author{
Sebastien Dubois, Ivana Rasovska, and Roland De Guio \\ INSA Graduate School of Science and Technology, 24 Boulevard de la Victoire, \\ 67084 Strasbourg Cedex, France \\ \{sebastien.dubois, ivana.rasovska, \\ roland.deguio\}@insa-strasbourg.fr
}

\begin{abstract}
There exist two kinds of problem resolution in the design of technical systems: the optimization resolution tools are appropriate for routine design whereas inventive resolution tools are appropriate for innovative and creative design. In general, the problems are tackled first by an optimization approach and, if no solution is found, inventive approaches are used to solve the problem. Previously, the Generalized System of Contradictions was defined to fit both kinds of problem resolution and to shift from optimization representation models to inventive ones. In this paper the transition is based on the identification of Generalized System of Contradictions out of Design of Experiments. The set of equations to resolve to automatically extract different kinds of contradictions out of a DoE's model is proposed.
\end{abstract}

Keywords: Design of Experiments, Generalized System of Contradictions, inventive problems.

\section{Introduction}

Designing could be described several ways, a common assumption is that it consists of a set of activities including problem resolution steps. Based on this definition, [1] proposes a classification of design into routine, innovative and creative where:

- Routine design proceeds within a totally defined state space, i.e. all design variables and their possible domains are known and the problem is one of possible instantiations.

- Innovative design proceeds within a space of known solutions which is extended by making variation or adaptations to existing designs, i.e. the domain of values of existing design variables is extended.

- Creative design defines a state space that may include an extended state space of possible solutions or creating a new state space.

Each of these different kinds of design involves specific resolution tools. In [2] some pertinent tools are presented as Constraints Solving Problems (CSP) for routine design; over-constrained CSP for innovative design and TRIZ resolution tools for 
creative design. More generally, all optimization tools are appropriate for routine design and inventive resolution tools are appropriate for both innovative and creative design. It is thus possible to define two families of resolution tools, those for optimization and those for invention. The invention resolution tools refer to defining a new state space either by extension - innovative design - either by creation of this space - creative design.

Using specific tools for specific kinds of problems seems logic but it is limited in the nature of the problem to solve which is rarely known at the beginning of the resolution and so one does not know what kind the problem is. In general, the problems are tackled by an optimization approach at first and, if no solution is found, inventive approaches are used to deal with the problem. This practice is rather difficult because of the complexity to shift from one approach to another. Once a model of the problem was built and the corresponding resolution tool was used to look for the solution, it is not trivial to reformulate the problem to set a new model when the considered approach did not work. To overcome this problem a link between optimization models and an inventive one has been proposed in [3]. This proposal is based on the definition of a new model, the Generalized System of Contradictions (GSC), based on TRIZ [4] models but extended to fit any situations than cannot be solved by optimization approaches. The shifting from optimization models to the Generalized System of Contradictions has been described in [2, 3] and will be briefly presented in section 2, which will mainly be dedicated to the definition of the GSC and of different kind of TRIZ-based contradictions. Till now this transition is based on the identification of a Generalized System of Contradictions out of models as Constraint Solving Problems (CSP) or Design of Experiments (DoE). The section 3 of this article will present the set of equations to resolve in order to automatically extract different kinds of contradictions out of a DoE. Then conclusion and perspectives will be proposed.

\section{Generalized System of Contradictions in Design of Experiments}

This section will briefly present the models of Generalized System of Contradictions and Design of Experiments to formulate the problems. Then the comparison of both models and the way to make them fit will be described. In a second part the required properties of different kinds of TRIZ-based contradiction, and in particular, the Generalized System of Contradictions model, will be described as they are necessary to enable the automatic extraction.

\subsection{Comparison of Models}

Design of Experiments [5] is an organized method for determining the relationship between factors (process variables Xs) affecting a process and the output of that process (response variables Y). Experimental design is a strategy to gather empirical knowledge, i.e. knowledge based on the analysis of experimental data and not on theoretical models. In an experiment, one or more process variables (or factors) are changed in order to observe the effects these changes have on one or more response variables (outputs). The factors are controlled parameters, whereas the outputs are measurable ones. 
One of the objectives of DoE is to obtain the most robust model with the minimum of experiments, which can be reached by the use of Taguchi's methods [6]. Taguchi recommended a two-step design process, robust design followed by tolerance design. Robust Design is a technique that reduces variation in a product by reducing the sensitivity of the design of the product to sources of variation rather than by controlling their sources. Tolerance Design is concerned with how much variation of the design and noise factors is permissible. Traditionally the operational steps for robust design are [7]:

- State a problem and its objective.

- List responses, control parameters, and sources of noise.

- Plan the experiment.

- Run experiment and predict improved parameter settings.

- Run confirmation experiment.

If the objective is not met, then it is necessary to go back to the second step. Otherwise, the improved design can be adopted.

Generalized System of Contradictions, illustrated on figure 1, is the generalization of the OTSM-TRIZ system of contradictions [8] based on the use of concepts, which are defined as logical assertions about values of the parameters [9]. Two concepts based on a set of action parameters satisfy two sets of evaluation parameters. The desired result is then the simultaneous satisfaction of the two sets of evaluation parameters. This generic model satisfies the following equivalence "a contradiction exists if and only if no solution can be found by optimization of a known model".

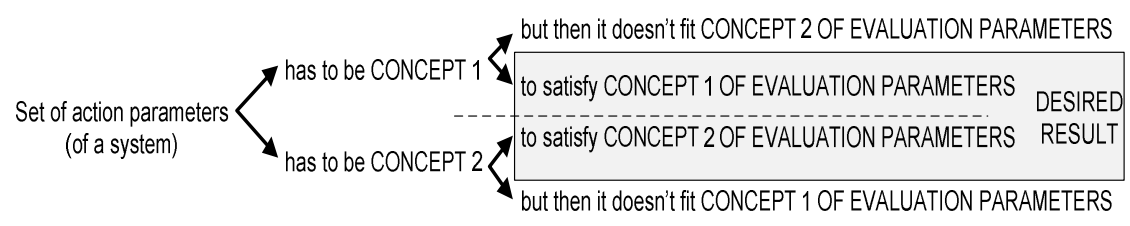

Fig. 1. Generalized System of Contradictions

Similarities and differences exist between the problem models of DoE and GSC. Even if the model of DoE is not explicitly defined in order to state problems, it is quite compatible with the model of Generalized System of Contradictions. The analogy between these two models is quite evident, as defined in table 1 . In the both cases, two categories of parameters are defined, those to evaluate the result and those to act on the system in order to reach the desired result.

Table 1. Comparison between the DoE and the GSC models

\begin{tabular}{lll}
\hline & Design of Experiments & $\begin{array}{c}\text { Generalized System of } \\
\text { Contradictions }\end{array}$ \\
\hline System model & Controlled Parameters & Action Parameters \\
Result evaluation & Measured Parameters & Evaluation Parameters \\
\hline
\end{tabular}


One of the assumptions for the definition of a GSC is that it only exists if no solution can be found among the realized experiments.

\subsection{Properties of the Generalized System of Contradictions Model}

The Generalized System of Contradictions is an extension of the models of contradiction defined in TRIZ [4]. The models of contradictions in TRIZ are based on a dialectical representation of the problems. This kind of problem formulation frames has proved its benefits for problem resolution in many domains. In [10] the limit of this model has been defined as not satisfying the equivalence between the two propositions: "the problem has no solution" and "a contradiction exists". To overcome this limitation the GSC has been defined. In this part the properties of this model will be presented as will be demonstrated that the other TRIZ based models are particular models that can be declined out of the GSC.

The properties of the Generalized System of Contradictions can be characterized by the set of definitions that enables the extraction of the GSC out of a DoE. Let one consider a DoE characterized by:

- a set of action parameters $\mathrm{X}=\left(\mathrm{x}_{0}, \mathrm{x}_{1}, \ldots, \mathrm{x}_{\mathrm{n}}\right)$

- a set of domains $\mathrm{D}=\left(\mathrm{D}_{0}, \mathrm{D}_{1}, \ldots, \mathrm{D}_{\mathrm{n}}\right)$ where $\mathrm{D}_{\mathrm{i}}$ defines the possible range of values for $\mathrm{x}_{\mathrm{i}}$

- a set of evaluation parameters $\mathrm{Y}=\left(\mathrm{y}_{0}, \mathrm{y}_{1}, \ldots, \mathrm{y}_{\mathrm{p}}\right)$ characterized by binary values, either 1 if $\mathrm{y}_{\mathrm{i}}$ is satisfied, 0 otherwise

- a set of experiments $E=\left(e_{0}, e_{1}, \ldots, e_{m}\right)$. An experiment $e_{i}$ is a particular instantiation of the action parameters: $\left(a_{i 1}, a_{i 2}, \ldots, a_{i n}\right)$, so that $a_{i j} \mapsto D_{j}$ and the induced values of evaluation parameters $\left(\mathrm{z}_{\mathrm{i} 1}, \mathrm{z}_{\mathrm{i} 2}, \ldots, \mathrm{z}_{\mathrm{ip}}\right)$, so that $\mathrm{z}_{\mathrm{ij}}=1$ if $\mathrm{y}_{\mathrm{j}}$ is satisfied in experiment $\mathrm{e}_{\mathrm{i}}, 0$ otherwise.

Let one defines that the objective is to satisfy all the evaluation parameters and that such a solution does not exist in the considered DoE, i.e. that no experiment enable the satisfaction of all the evaluation parameters.

Identifying a Generalized System of Contradictions in such a DoE is looking for:

- Three sets of evaluation parameters $\mathrm{Y}_{0}, \mathrm{Y}_{1}$ and $\mathrm{Y}_{2}$, such as $\mathrm{Y}_{0} \cap \mathrm{Y}_{1}=\varnothing, \mathrm{Y}_{1} \cap \mathrm{Y}_{2}=\varnothing$, $\mathrm{Y}_{0} \cap \mathrm{Y}_{2}=\varnothing, \mathrm{Y}_{0} \cup \mathrm{Y}_{1} \cup \mathrm{Y}_{2}=\mathrm{Y}, \mathrm{Y}_{1} \neq \varnothing$ and $\mathrm{Y}_{2} \neq \varnothing$.

- Three sets of experiments $\mathrm{E}_{0}, \mathrm{E}_{1}$ and $\mathrm{E}_{2}: \mathrm{E}_{0} \cap \mathrm{E}_{1}=\varnothing, \mathrm{E}_{1} \cap \mathrm{E}_{2}=\varnothing, \mathrm{E}_{0} \cap \mathrm{E}_{2}=\varnothing$, $\mathrm{E}_{0} \cup \mathrm{E}_{1} \cup \mathrm{E}_{2}=\mathrm{E}, \mathrm{E}_{1} \neq \varnothing$ and $\mathrm{E}_{2} \neq \varnothing$. Moreover

- $\mathrm{E}_{1}$ is a set of experiments for which all the evaluation parameters of $\mathrm{Y}_{1}$ are satisfied.

- $\mathrm{E}_{2}$ is a set of experiments for which all the evaluation parameters of $\mathrm{Y}_{2}$ are satisfied.

Such a definition gives the way to reorganize the DoE table by permutations of the rows and of the columns in order to group the previously defined Ei and Yi (cf. Table 2) 
Table 2. GSC representation in a DoE

\begin{tabular}{|c|c|c|c|c|c|c|c|c|c|c|}
\hline & & & & \multicolumn{2}{|r|}{$\overline{\mathrm{Y1}}$} & \multicolumn{2}{|c|}{$\bar{Y} 2$} & \multicolumn{2}{|c|}{$\bar{Y}$} \\
\hline & & $\mathrm{x} 1$ & $x 2$ & $\mathrm{xn}$ & ys & $\ldots$ & $\mathrm{yu}$ & $\mathrm{yv}$ & $y w$ & $y h$ \\
\hline \multirow{3}{*}{$\mathrm{E} 1$} & ei & ai1 & ai2 & ain & \multirow{3}{*}{\multicolumn{2}{|c|}{$\begin{array}{c}E 1 \times Y 1: \\
z i j=1\end{array}$}} & \multirow{3}{*}{\multicolumn{2}{|c|}{$\begin{array}{l}\forall \text { ei } \in E 1 \\
\text { ei } \times Y 2: \\
\exists j / z i j=0\end{array}$}} & & \\
\hline & & & & & & & & & & \\
\hline & ej & aj1 & aj2 & ajn & & & & & & \\
\hline \multirow{3}{*}{ E2 } & ek & ak1 & ak2 & akn & \multirow{3}{*}{\multicolumn{2}{|c|}{$\begin{array}{c}\forall \text { ei } \in \text { E2 } \\
\text { ei } \times Y 1: \\
\exists j / z i j=0\end{array}$}} & \multirow{3}{*}{\multicolumn{2}{|c|}{$\begin{array}{c}E 2 \times Y 2: \\
z i j=1\end{array}$}} & & \\
\hline & & & & & & & & & & \\
\hline & el & al1 & $\mathrm{al} 2$ & aln & & & & & & \\
\hline \multirow{3}{*}{ EO } & eq & $\mathrm{aq1}$ & $\mathrm{aq} 2$ & aqn & & & & & & \\
\hline & & & & & & & & & & \\
\hline & er & ar1 & ar2 & arn & & & & & & \\
\hline
\end{tabular}

Based on this definition, it is possible to show that existing TRIZ-based models are particular models derivated from the GSC. The classical TRIZ contradictions are a particular case of GSC (see table 3), where:

- $\operatorname{card} \mathrm{Y}_{\mathrm{i}, \mathrm{i}=\{1,2\}=1}$

- $\exists\left(\mathrm{x}_{\mathrm{i}}, \mathrm{v}_{1}, \mathrm{v}_{2}\right) \in\left(\mathrm{X}, \mathrm{D}_{\mathrm{i}}, \mathrm{D}_{\mathrm{i}}\right) /\left(\mathrm{x}_{\mathrm{i}}=\mathrm{v}_{1} \Rightarrow\left(\mathrm{Y}_{1}, \mathrm{Y}_{2}\right)=(1,0)\right) \wedge\left(\mathrm{x}_{\mathrm{i}}=\mathrm{v}_{2} \Rightarrow\left(\mathrm{Y}_{1}, \mathrm{Y}_{2}\right)=(0,1)\right)$

Table 3. Classical TRIZ contradiction

\begin{tabular}{|c|c|c|c|c|c|c|c|c|}
\hline & & & & \multirow[t]{2}{*}{$\overline{Y 1}$} & \multirow{2}{*}{$\begin{array}{l}\mathrm{Y} 2 \\
\mathrm{yu}\end{array}$} & \multicolumn{2}{|c|}{ YO } \\
\hline & & $\mathrm{x} 1$ & $x g$ & $x n$ & & & $\mathrm{yw}$ & $\mathrm{yh}$ \\
\hline \multirow{3}{*}{ E1 } & ei & ai1 & \multirow{3}{*}{$\mathrm{v} 1$} & ain & \multirow{3}{*}{1} & \multirow{3}{*}{0} & & \\
\hline & $\ldots$ & & & & & & & \\
\hline & $\mathrm{ej}$ & aj1 & & ajn & & & & \\
\hline \multirow{3}{*}{ E2 } & ek & ak1 & \multirow{3}{*}{ v2 } & akn & \multirow{3}{*}{0} & \multirow{3}{*}{1} & & \\
\hline & ... & & & & & & & \\
\hline & \begin{tabular}{|l|}
$\mathrm{el}$ \\
\end{tabular} & al1 & & aln & & & & \\
\hline \multirow{3}{*}{ E0 } & eq & aq1 & aq2 & aqn & & & & \\
\hline & ${ }_{n}$ & & & & & & & \\
\hline & er & ar1 & ar2 & arn & & & & \\
\hline
\end{tabular}

The system of contradictions used in [11] is also a particular case of the GSC where, (cf. table 4):

- $\operatorname{card} \mathrm{Y}_{1}=$ card $\mathrm{Y}_{2}$

- $\exists\left(\mathrm{x}_{\mathrm{i}}, \mathrm{v}_{1}, \mathrm{v}_{2}\right) \in\left(\mathrm{X}, \mathrm{D}_{\mathrm{i}}, \mathrm{D}_{\mathrm{i}}\right) /\left(\mathrm{x}_{\mathrm{i}}=\mathrm{v} 1 \Rightarrow\left(\mathrm{Y}_{1}, \mathrm{Y}_{2}\right)=(1,0)\right) \wedge\left(\mathrm{x}_{\mathrm{i}}=\mathrm{v}_{2} \Rightarrow\left(\mathrm{Y}_{1}, \mathrm{Y}_{2}\right)=(0,1)\right)$ 
Table 4. System of contradictions from [11]

\begin{tabular}{|c|c|c|c|c|c|c|c|c|c|c|}
\hline & & & & \multicolumn{2}{|c|}{$\overline{\mathrm{Y1}}$} & \multicolumn{2}{|r|}{$\bar{Y} 2$} & \multicolumn{2}{|c|}{$\overline{Y O}$} \\
\hline & & $\mathbf{x 1}$ & $x g$ & $\mathrm{xn}$ & ys & $\mathrm{yt}$ & $\mathrm{yu}$ & yv & $\mathrm{yw}$ & \begin{tabular}{c|c|}
$\ldots y h$ \\
\end{tabular} \\
\hline \multirow{3}{*}{ E1 } & ei & ai1 & \multirow{3}{*}{ v1 } & ain & \multirow{3}{*}{\multicolumn{2}{|c|}{$\begin{array}{c}\text { E1XY1: } \\
z i j=1\end{array}$}} & \multirow{3}{*}{\multicolumn{2}{|c|}{$\begin{array}{l}\forall \text { ei } \in E 1 \\
\text { ei } \times \text { Y2: } \\
\exists j / z i j=0\end{array}$}} & & \\
\hline & & & & & & & & & & \\
\hline & ej & aj1 & & ajn & & & & & & \\
\hline \multirow{3}{*}{ E2 } & ek & ak1 & \multirow{3}{*}{ v2 } & akn & \multirow{3}{*}{\multicolumn{2}{|c|}{$\begin{array}{l}\forall \text { ei } \in \text { E2 } \\
\text { ei } \times Y 1: \\
\exists j / z i j=0\end{array}$}} & \multirow{3}{*}{\multicolumn{2}{|c|}{$\begin{array}{c}E 2 \times Y 2: \\
z i j=1\end{array}$}} & & \\
\hline & & & & & & & & & & \\
\hline & el & al1 & & aln & & & & & & \\
\hline \multirow{3}{*}{ E0 } & eq & aq1 & aq2 & aqn & & & & & & \\
\hline & & & & & & & & & & \\
\hline & er & ar1 & ar2 & arn & & & & & & \\
\hline
\end{tabular}

These particularities show the generic aspect of the GSC enabling to define, under certain conditions existing TRIZ-based contradictions. The classical TRIZ model of contradiction is easier to solve as it is easier to interpret for a human expert of the domain, it is more significant. Whereas the GSC is more difficult to interpret and so to solve but it exists in any problematic situation for which no solution is known, this contradiction is illustrated on Fig 2.

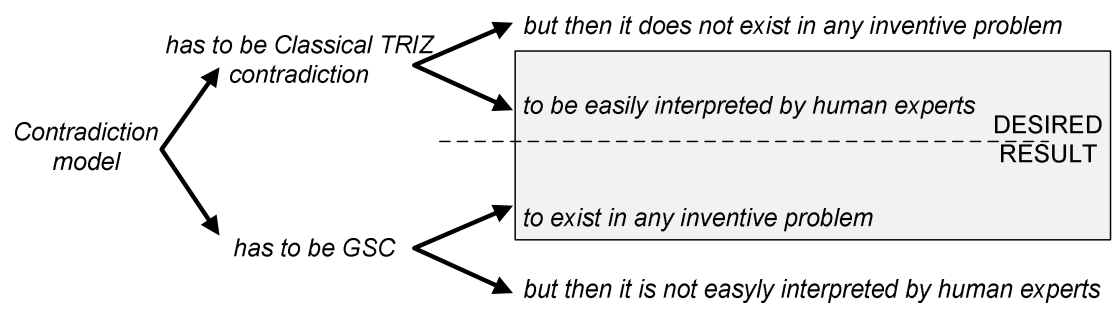

Fig. 2. Contradiction of the model to be used for inventive problems

The next part will be dedicated to the definition of the algorithms to extract those different levels of contradictions.

\section{Extraction of the GSC in a DoE}

The analysis and definition of the equations to solve in order to extract the three sets of $Y_{i}$ and the three sets of $E_{i}$ out of the DoE will be presented in this part, as will be presented the way to extract the other previously presented TRIZ-based contradictions. The permutation of the rows and columns can be obtained by similarity research algorithms such as quasi-seriation ones. Identifying a Generalized System of Contradictions is making a quasi-seriation of two classes where the residuals define $Y_{0}$ and the two classes define $\mathrm{Y}_{1}$ and $\mathrm{Y}_{2}$. 
The extraction of a GSC is looking for the solution of the following set of equations:

$$
\begin{aligned}
& \sum_{i j}=1 \text { or } 0 \\
& \sum_{i / e_{i} \in E_{k} ; j / y_{j} \notin Y_{k}} \overline{z_{i j}} \geq 1 \\
& \forall\left(e_{i}, y_{j}\right) \in E_{k \neq 0} \times Y_{k \neq 0} ; z_{i j}=1 \\
& \forall\left(e_{i}, y_{j}\right) \in E_{0} \times Y_{k \neq 0}: \sum_{j / y_{j} \in Y_{k}} \overline{z_{i j}} \geq 1 \\
& \forall\left(e_{i}, y_{j}\right) \in E_{k \neq 0} \times Y_{0}: \sum_{i / e_{i} \in E_{k}} \overline{z_{i j}} \geq 1
\end{aligned}
$$

To extract classical TRIZ contradictions the set of equations to resolve is:

$$
\begin{aligned}
& z_{i j}=1 \text { or } 0 \\
& \sum_{i / e_{i} \in E_{k} ; j / y_{j} \notin Y_{k}} z_{i j}=0 \\
& \forall\left(e_{i}, y_{j}\right) \in E_{k \neq 0} \times Y_{k \neq 0} ; z_{i j}=1 \\
& \forall e_{i} \in E_{0}: z_{i j, j / y_{j} \in Y_{1}}+z_{i k, k / y_{k} \in Y_{2}} \leq 1 \\
& \forall e_{i} \in E_{1}: a_{i j}=v_{1} \\
& \forall e_{i} \in E_{2}: a_{i j}=v_{2} \\
& v_{1} \neq v_{2}
\end{aligned}
$$

Whereas the extraction of contradictions from [11] is based on the resolution of the following set of equations:

$$
\begin{aligned}
& \sum_{i j}=1 \text { or } 0 \\
& \sum_{i / e_{i} \in E_{k} ; j / y_{j} \notin Y_{k}} \overline{z_{i j}} \geq 1 \\
& \forall\left(e_{i}, y_{j}\right) \in E_{k \neq 0} \times Y_{k \neq 0} ; z_{i j}=1 \\
& \forall\left(e_{i}, y_{j}\right) \in E_{0} \times Y_{k \neq 0}: \sum_{j / y_{j} \in Y_{k}} \overline{z_{i j}} \geq 1 \\
& \forall\left(e_{i}, y_{j}\right) \in E_{k \neq 0} \times Y_{0}: \sum_{i / e_{i} \in E_{k}} \overline{z_{i j}} \geq 1 \\
& \forall e_{i} \in E_{1}: a_{i j}=v_{1} \\
& \forall e_{i} \in E_{2}: a_{i j}=v_{2} \\
& v_{1} \neq v_{2}
\end{aligned}
$$


The differences between these sets of equations to resolve in order to extract the different kinds of TRIZ-based contradictions show that the classical TRIZ system of contradiction is the most constrained one; such a contradiction has the limitation to not fit the equivalence (no solution) $\Leftrightarrow$ (a contradiction exists). Even if the system of contradictions from [11] is less constrained, it does not fit this equivalence too.

These descriptions of the existing TRIZ-based contradictions show that it is possible to define more or less generic contradictions; our interest for the GSC is based on the satisfaction of the previously defined equivalence.

Concerning the algorithm to solve the set of equations defining a GSC; several questions have to be explored and answered.

In fact there are several ways to build a GSC and moreover to choose the GSC that has to be solved firstly. Two kinds of strategies could be defined:

- Solving a problem means understanding the limits of the model used to represent the problem, and so being able to change this model [12]. Thus, to propose an efficient model change, it is meaningful to build a model based on the biggest amount of knowledge about the problematic situation. Such a strategy leads to the building of a GSC minimizing the cardinality of $\mathrm{E}_{0}$. Such a GSC is based on more experiments of the problem model; this GSC could be referred as exhaustive GSC.

- To solve a contradiction also means to satisfy all evaluation parameters implied in the contradiction, whereas solving the problem means satisfying all evaluation parameters. If one aims at solving the problem in a "one-shot" contradiction resolution, the contradiction model has to be based on all the evaluation parameters. Such a GSC will be built by defining a GSC so that $Y=Y_{1} \cup Y_{2}$ and could be called an efficient GSC.

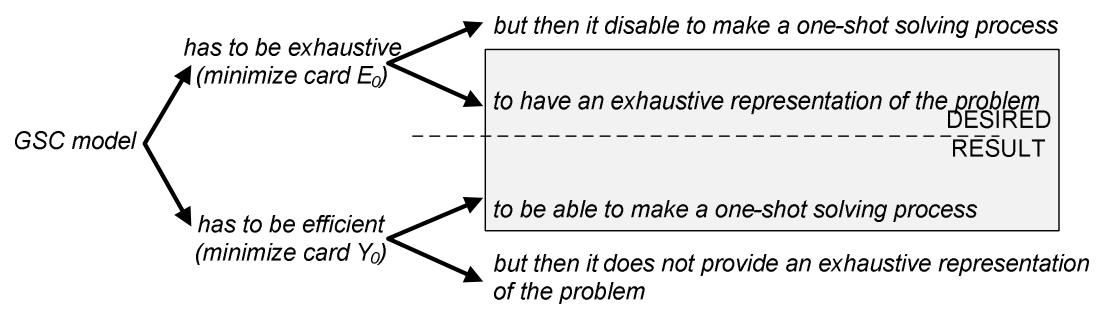

Fig. 3. Contradiction of the strategy to build the GSC

A way to solve the contradiction presented in figure 3 is to build an efficient GSC, enabling to go towards a "one-shot solving" process and propose a discriminatory and exhaustive definition of the concepts used in the GSC [9]. Once the way to build the GSC is defined, several GSC could fit the constraints. The choice could then aim at resolving the contradiction presented on figure 1 and so defining a GSC that will be more comprehensive for human domain experts, a meaningful GSC. A last hypothesis is to build a GSC in accordance with its resolution, either a GSC that will be easier to solve, or a GSC that will leads to the more robust solutions. Currently these different hypotheses have to be tested in order to check the most useful way to define the GSC in order to solve the problem. 


\section{Conclusion}

The Generalized System of Contradictions was described as a model that fits both optimization and inventive problem resolution methods and tools. The fact that exist different models of contradictions illustrating different levels of contradictions shows the generic aspect of the GSC, as it enables to define, under certain conditions, those existing TRIZ-based contradictions. The comparison with Design of Experiments model was presented in order to introduce the automatic extraction of GSC from the solutionless DoE's problem model. To extract different models of contradictions three sets of equations was proposed to be solved. The differences between these sets of equations show the existence of less or more generic contradictions and underline our interest for the GSC to satisfy the equivalence that a problem contradiction exists if and only if there is no solution of the problem as the criterion for retained contradictions. Two strategies to build a GSC model and consequently to solve it were proposed according to the exhaustiveness of the problem model and the efficiency of its resolution. The testing of these strategies still remains as well as the definition of the most useful way to define the GSC in order to be meaningful for human experts and in order to be efficiently solvable. The work dedicated to the identification of the GSC can be based on the use of similarity research algorithm such as quasi-seriation ones. There are plenty of existing tools to facilitate this part.

\section{References}

1. Rosenman, M.A., Gero, J.S.: Creativity in design using a design prototype approach. In: Gero, J.S., Maher, M.L. (eds.) Modeling Creativity and Knowledge-Based Creative Design, pp. 111-138. Lawrence Erlbaum Associates, Inc., Mahwah (1993)

2. Dubois, S., Rasovska, I., De Guio, R.: Comparison of non solvable problem solving principles issued from CSP and TRIZ. In: Cascini, G. (ed.) IFIP International Federation for Information Processing, vol. 277, pp. 83-94. Springer, Boston (2008)

3. Eltzer, T., De Guio, R.: Constraint based modelling as a mean to link dialectical thinking and corporate data. Application to the Design of Experiments. In: 2nd IFIP Working Conference on Computer Aided Innovation. Springer, Brighton (2007)

4. Altshuller, G.S.: Creativity as an Exact Science. Gordon and Breach, New York (1988)

5. Montgomery, D.C.: Design and Analysis of Experiments. Wiley-Interscience, Hoboken (2004)

6. Roy, R.K.: Design of Experiments Using The Taguchi Approach: 16 Steps to Product and Process Improvement. Wiley-Interscience, Hoboken (2001)

7. Buyske, S.: Advanced Design of Experiments. Rutgers University, Piscataway (2001)

8. Khomenko, N., De Guio, R., Lelait, L., Kaikov, I.: A framework for OTSM-TRIZ-based computer support to be used in complex problem management. International Journal of Computer Applications in Technology 30, 88-104 (2007)

9. Dubois, S., Rasovska, I., De Guio, R.: Interpretation of a General Model for Inventive Problems, the Generalized System of Contradictions. In: Roy, R., Shehab, E. (eds.) Competitive Design. Proceedings of the 19th CIRP Design Conference, pp. 271-276. Cranfield University Press, Cranfield (2009) 
10. Dubois, S., Eltzer, T., De Guio, R.: A dialectical based model coherent with inventive problems and optimization problems. Computers in Industry Accepted, to be published (2009)

11. Cavallucci, D., Rousselot, F., Zanni, C.: Representing and selecting problems through contradiction clouds. In: Cascini, G. (ed.) Computer-Aided Innovation (CAI), vol. 277, pp. 43-56. Springer, Boston (2008)

12. Rasovska, I., Dubois, S., De Guio, R.: Mechanisms of Model Change in Optimization and Inventive Problem Solving Methods. In: International Conference on Engineering Design, ICED 2009, Stanford, CA, USA (2009) 